\title{
The Detection Performance of the Dual-Sequence-Frequency-Hopping Signal via Stochastic Resonance Processing under Color Noise
}

\author{
Guangkai LIU ${ }^{1}$, Yanmei KANG ${ }^{2}$, Houde $Q_{U A N}{ }^{1}$, Huixian SUN ${ }^{1}$, Peizhang CUI ${ }^{1}$, Chao GUO ${ }^{3}$ \\ ${ }^{1}$ Dept. of Electronics and Optical Engineering, Army Engineering University, Shijiazhuang, China \\ 2 Dept. of Applied Mathematics, School of Mathematics and Statistics, Xi' an Jiaotong University, Xi' an, China \\ 3 The Troop of 32125 , Jinan, China \\ dreamer_gk@163.com
}

Submitted May 22, 2019 / Accepted July 16, 2019

\begin{abstract}
Can the Dual-Sequence-Frequency-Hopping (DSFH) as a military emergency communication mode work under strong color noise? And is there any detection improvement of the DSFH signal via stochastic resonance (SR) processing under color noise? To deal with this problem, we analyze the physical feature of the DSFH signal. Firstly, the signal models of transmission, reception and the intermediate frequency $(I F)$ are constructed. And the scale transaction is used to adjust the IF signal to fit the SR. Secondly, the non-markovian Langevin Equation (LE) is transformed into a markovian one by expand the 1-D LE to a 2-D one. Thirdly, the non-autonomous Fokker-Plank Equation (FPE) is transformed into an autonomous one by assuming that the SR transition of magnetic particles is instantaneous and introducing the decision time. Therefore, the analytical periodic steady solution of the probability density function (PDF) with the parameter of the correlation time of the color noise is obtained. Finally, the detection probability, false alarm probability and Receiver Operating Characteristics (ROC) curve are obtained, under the criterion of the maximum a posterior probability (MAP). Theoretical and simulation results show as below: 1) whether the DSFH can work under strong color noise is decided by the correlation time of the color noise; 2) when the power intensity of the color noise is constant, the smaller the correlation time with the bigger local SNR, the greater PDF difference of the SR output under two hypothesis, leading to better detection performance.
\end{abstract}

\section{Keywords}

Dual-Sequence Frequency Hopping, stochastic resonance, detection performance, color noise

\section{Introduction}

The Dual Sequence Frequency Hopping (DSFH) communication mode draw lessons from "the medium is the mes- sage" [1], [2]. The chosen medium is the communication channel, at the same time the unchosen one is the dual channel controlled by two PN sequence represented of the symbol 0 or 1 . Then the receptive symbol is decided by whether the channel is occupied or not [3-5]. The DSFH is one of the covert communication, which brings some anti-interference ability congenitally. But errors occur to the energy detector when the dual channel is occupied by strong noise. What is more, the color noise is the most actual noise. How to raise the detection performance of the DSFH's signal under color noise is the most factual problem. Some researchers apply the stochastic resonance (SR) to detect signals under color noise. SR as a nonlinear detector can transact parts of the noise energy to the signal [6-8], raising the detection performance lower than the threshold [9], which can expand the DSFH's application further. Peter Hanggi does researches on the effects of the color noise on SR, and conclude that color noise can enhance SR [10]. M.A. Fuentes and Raul Toral study the effects of non-Gaussian noises bringing to $\mathrm{SR}$, use the path-integral method and obtain the periodic solution of the Fokker-Planck Equation (FPE) [11], [12]. Jia et al. investigate the effects of color noise on SR by using the unified colored noise approximation and the theory of signal-to-noise ratio (SNR) in the adiabatic limit [13]. Xu et al. study the relationship between the correlation time and the coherent bandwidth, then obtain the solution of the SNR gain and the characteristic time [14]. However the effects of color noise on DSFH signal processing have not yet explored sufficiently.

In this paper, we will deal with the DSFH signal processing by a bi-stable SR system subjected to the color noise. Via introducing the decision time, the periodic steady-state solution of FPE is obtained. What is more, the detection probability and the Receiver Operating Characteristic (ROC) are obtained. The paper is organized as follows: In Sec. 2, we investigate the model of the transmitted and received DSFH signal, and the SR processing of the received signal. Section 3 is devoted to the studies of the detection performance 
by the periodic steady-state solution of FPE via introducing the decision time. In Sec. 4, the numerical simulations verifies the theory. The last section draws some conclusions.

\section{The System Model of the DSFH}

\subsection{The Transmitted Signal of the DSFH}

The communication and dual carriers controlled by PN sequences are chosen by the transmitted symbol in the DSFH mode, described as Fig. 1.

The channel 0 and 1 are respectively the carrier $f_{0, n}$ and $f_{1, n}$ controlled by PN sequences $\mathrm{FS}_{0}$ and $\mathrm{FS}_{1}$. At the time of $t$, the sine carrier $s_{0}(t)$ with frequency of $f_{0, n}$ is transmitted if the transmitted symbol is 0 . Otherwise the sine carrier $s_{1}(t)$ represented of symbol 1 is transmitted. The final transmitted signal $s(t)$ of DSFH is the combination of the $s_{0}(t)$ and the $f_{0, n}$, after the channel switch.

Assume that the transmitted data is $\mathbf{b}=$ $(\cdots, 1,0,1,0, \cdots)$, then the transmitted signal is the sine carrier with frequency of $\left(\cdots, f_{2}, f_{4}, f_{3}, f_{1}, \cdots\right)$. So the radio frequency (RF) signal of DSFH can be represented as

$$
\begin{aligned}
s(t)= & \cos \left[2 \pi f_{i, n}\left(t-n T_{\mathrm{s}}\right)+\varphi\right] \\
\cdot & {\left[\varepsilon\left(t-n T_{\mathrm{S}}\right)-\varepsilon\left(t-(n+1) T_{\mathrm{s}}\right)\right] }
\end{aligned}
$$

where $T_{\mathrm{s}}$ is the hop duration, $\varepsilon(t)$ is the step signal, $f_{i, n}$ is the radio frequency of the $n$th hop, and $f_{i, n}=\left\{\begin{array}{l}f_{0, n}, \text { transmitted symbol is } 0 \\ f_{1, n}, \text { transmitted symbol is } 1\end{array} f_{0, n} \neq f_{1, n}\right.$.

\subsection{The Receptive Signal of the DSFH}

The super-heterodyne receiver is adopted in DSFH, as depicted in Fig. 2.

The signal $r(t)$ is receipted under color noise at the RF front-end. Then be mixed with the super-heterodyne carriers controlled by two PN sequences. Subsequently the analog beat signal as the intermediate frequency (IF) signal be converted to the digital by the $\mathrm{A} / \mathrm{D}$ convertor. The frequency and waveform of the IF signal obtained by the two receptive branches are the same, which can be descripted as

$$
\begin{aligned}
s(t)= & \cos \left[2 \pi f_{0}\left(t-n T_{\mathrm{S}}\right)+\varphi\right] \\
\cdot & {\left[\varepsilon\left(t-n T_{\mathrm{S}}\right)-\varepsilon\left(t-(n+1) T_{\mathrm{S}}\right)\right] }
\end{aligned}
$$

where $f_{0}$ is the pre-set frequency of the IF signal.

With the reason that the $f_{0}$ and the sample frequency $f_{\mathrm{s}}$ are set as $1 \mathrm{kHz}$ and $200 \mathrm{kHz}$, the IF signal can be viewed as low frequency signal, which can be processed by Low-pass Filter (LF). Then the scale transact unit (ST) adapt the parameters of IF signal to fit the need of SR. The output of the SR is the test statistic, and after sampling and decision the receptive symbol of single branch is obtained. Assuming the synchronization is completed, the receptive symbols of two branches are the receptive symbols after combination.

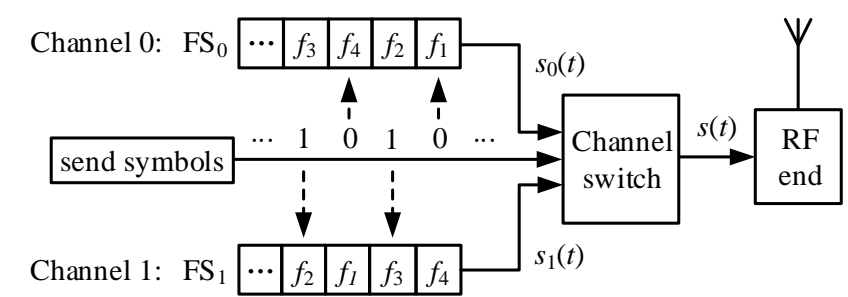

Fig. 1. The transmitted structure of the DSFH.

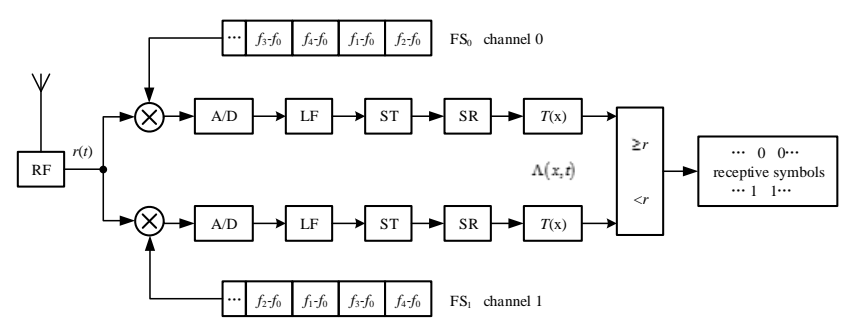

Fig. 2. The received structure of the DSFH.

The noise processed by LF becomes color and nonGaussian, whose spectrum can be assumed as a Lorentzian form. So the background noise of the receptive DSFH signal via SR processing is color. Next we will analyze the improvement of the color noise bringing to the detection of the DSFH signal detection.

\subsection{The SR Processing of the Receptive Signal}

The IF signal of the two receptive branches is the sine wave as depicted in (2). The overdamped bi-stable SR enforced by sine wave $A \cos \left(\omega_{0} t+\varphi\right)$ and color noise $Q(t)$ can be depicted as

$$
\frac{\mathrm{d} x}{\mathrm{~d} t}=a x-b x^{3}+A \cos \left(\omega_{0} t+\varphi\right)+Q(t)
$$

where $a$ and $b$ are the SR parameters, $A \cos \left(\omega_{0} t+\varphi\right)$ is the periodic signal, $Q(t)$ is the color noise, whose spectrum can be assumed as a Lorentzian form like $S(\omega)=\frac{2 D}{1+\tau^{2} \omega^{2}}$, and the corresponding correlation function is $\left\langle Q(t) Q\left(t^{\prime}\right)\right\rangle=\frac{D}{\tau} \exp \left(-\frac{\left|t-t^{\prime}\right|}{\tau}\right), \tau$ is the correlation time of the color noise.

With the reason that SR unite can only settle the small signal with small frequency and amplitude, so the IF signal with large frequency and amplitude must be transacted to small one, which is done by the ST unite. Therefore we introduce the variable substitution [15] $z=x \sqrt{\frac{b}{a}}, \tau=a t$, equation (3) can be transacted to

$$
\frac{\mathrm{d} z}{\mathrm{~d} t}=z-z^{3}+\sqrt{\frac{b}{a^{3}}} A \cos \left(\frac{\omega_{0}}{a} \tau+\varphi\right)+\sqrt{\frac{2 D b}{a^{2}}} Q(\tau) .
$$

So, the frequency scale transacted equation is $\frac{\omega_{0}}{a}=2 \pi f$, where the frequency is transacted to the $\frac{1}{a}$ times of the original. The amplitude scale transacted equation is $A_{0}=\sqrt{\frac{b}{a^{3}}} A$. When parameter of $a$ is large enough and $b$ is 
small enough, the large IF signal can be transacted to small signal after ST. At the same time, the noise intensity becomes $D_{0}=\sqrt{\frac{2 D b}{a^{2}}}$. In order to accord with the habit of representation of variables, equation (4) is rewritten as

$$
\frac{\mathrm{d} x}{\mathrm{~d} t}=x-x^{3}+\sqrt{\frac{b}{a^{3}}} A \cos \left(\frac{\omega_{0}}{a} t+\varphi\right)+\sqrt{\frac{2 D b}{a^{2}}} Q(t)
$$

Due to the correlation time of $Q(t)$, equation (5) is not Markov process. But we can extend it into two dimensional space, which can be described by Markov process as

$$
\begin{gathered}
\frac{\mathrm{d} x}{\mathrm{~d} t}=C(x)+\sqrt{\frac{2 D b}{a^{2}} y,} \\
\frac{\mathrm{d} y}{\mathrm{~d} y}=-\frac{1}{\tau} y+\frac{1}{\tau} \Gamma(t)
\end{gathered}
$$

where $C(x)=x-x^{3}+\sqrt{\frac{b}{a^{3}}} A \cos \left(\frac{\omega_{0}}{a} t+\varphi\right), \Gamma(t)$ is the Gaussian white noise with intensity of $D$.

Therefore the corresponding FPE of (6) can be descripted as

$$
\begin{aligned}
& c \frac{\partial \rho(x, y, t)}{\partial t}=-\frac{\partial}{\partial x}\left\{\left[C(x)+\sqrt{\frac{2 D b}{a^{2}} y}\right] \rho(x, y, t)\right\} \\
&+\frac{\partial}{\partial y}\left[\frac{1}{\tau} y \rho(x, y, t)\right]+D^{\prime} \frac{\partial^{2}}{\partial y^{2}}[\rho(x, y, t)]
\end{aligned}
$$

where $D^{\prime}=\frac{D}{\tau^{2}}, a$ and $b$ are the parameters of SR, $\rho(x, y, t)$ is the PDF of the particle locating $(x, y)$ at time $t$.

Owing to the non-autonomous item $\cos \left(\frac{\omega_{0}}{a} t+\varphi\right)$ $\rho(x, y, t)$, equation (7) can't be solved by steady-state solution, and can't be solved by any exact solution [16]. So with the characteristic of the electromagnetic particle, we assume that the SR processing is completed transiently, neglecting the transacting time from the unsteady state to the steady. Then we introduce the decision time $t_{0}$, and the nonautonomous item $\cos \left(\frac{\omega_{0}}{a} t+\varphi\right) \rho(x, y, t)$ can be viewed as time-drift coefficient $\cos \left(\frac{\omega_{0}}{a} t_{0}+\varphi\right) \rho(x, t)$ depended on $t_{0}$. Therefore the non-autonomous equation is transacted to an autonomous one, which can be solved easier.

We use the Taylor expansion, and derive the approximate periodic steady solution of (7) as [16]

$$
\begin{aligned}
\rho\left(x \mid t_{0}\right)= & N \sqrt{\frac{4 \pi D^{2} b}{a^{2} \tau\left[1-\tau c^{\prime}\left(x, t_{0}\right)\right]}} \\
& \cdot\left\{1+\frac{5 D b \tau^{3}\left[c^{\prime \prime}\left(x, t_{0}\right)\right]^{2}}{12 a^{2}\left[1-\tau c^{\prime}\left(x, t_{0}\right)\right]^{5}}\right\} \\
& \cdot\left\{\left[\frac{\sqrt{2 D b}}{a}-\frac{\tau \sqrt{2 D b} c^{\prime}\left(x, t_{0}\right)}{a}\right]\right\}^{\frac{3}{2}} \\
& \cdot \exp \left[\frac{1}{D} \int c\left(x, t_{0}\right) d x-\frac{1}{2 D} \tau c^{2}\left(x, t_{0}\right)\right]
\end{aligned}
$$

where $D$ is the intensity of color noise, $\tau$ is the correlation time of the color noise, $a$ and $b$ are the parameters of SR, $\omega_{0}$ is the frequency of IF signal, $t_{0}$ is the decision time, $N$ is the normalization constant,

$c\left(x, t_{0}\right)= \begin{cases}x-x^{3} & , \mathrm{H}_{0} \\ x-x^{3} \pm \sqrt{\frac{b}{a^{3}}} A \cos \left(\frac{\omega_{0}}{a} t_{0}+\varphi\right) & , \mathrm{H}_{1}\end{cases}$

is the drift force of the SR varying with the input signal.

As descripted of (8), the sine wave at decision time $t_{0}$ equals to the linear drift force pulling the particles to the bilateral well, which increase the pause probability at the bilateral well. The positive drift force pulls particles to the positive well, while the negative drift force pulls particles to the negative well. Therefore owing to the periodic sine wave and the symmetry of particles of SR, the output probability of particles has symmetry properties. At the same time, different decision time decides different drift force because of the periodic properties of sine wave. The drift force reaches the largest when the decision time is the peak or valley of the sine wave, leading to that the pause time reaches the longest and the output probability reaches the largest.

\section{The Detection Performance}

The case does not exit that there are both signals at two branches of DSFH. For the single branch, the hypothesis testing problem is

$$
\left\{\begin{array}{lc}
\mathrm{H}_{0}: & y(t)=n(t) \\
\mathrm{H}_{1}: & y(t)=x(t)+n(t)
\end{array}\right.
$$

where $x(t)$ and $n(t)$ are the signal and the noise output of the SR. Assuming that $t_{0}$ is the best decision time, so it comes down to the hypothesis testing problem with the parameter $t_{0}$. So the conditional probability density under two hypotheses becomes to (10), where $t_{0}$ is the best decision time, $x_{1}$ and $x_{2}$ are the value range of $x$, and the other parameters are the same as in (8). 


$$
\left\{\begin{aligned}
P\left(x \mid \mathrm{H}_{0}, t_{0}\right)= & \rho\left(x, t_{0} \mid \mathrm{H}_{0}\right)=N_{0} \sqrt{\frac{4 \pi D^{2} b}{a^{2} \tau\left[1-\tau\left(1-3 x^{2}\right)\right]}} \cdot\left\{1+\frac{15 D b \tau^{3} x^{2}}{a^{2}\left[1-\tau\left(1-3 x^{2}\right)\right]^{5}}\right\} \\
& \cdot\left\{\left[\frac{\sqrt{2 D b}}{a}-\frac{\tau \sqrt{2 D b}\left(1-3 x^{2}\right)}{a}\right]\right\}^{\frac{3}{2}} \cdot \exp \left[\frac{1}{D} \int_{x_{1}}^{x_{2}}\left(x-x^{3}\right) \mathrm{d} x-\frac{1}{2 D} \tau\left(x-x^{3}\right)^{2}\right] \\
P\left(x \mid \mathrm{H}_{1}, t_{0}\right)= & \rho\left(x, t_{0} \mid \mathrm{H}_{1}\right)=N_{1} \sqrt{\frac{4 \pi D^{2} b}{a^{2} \tau\left[1-\tau\left(1-3 x^{2}\right)\right]}}\left\{1+\frac{15 D b \tau^{3} x^{2}}{a^{2}\left[1-\tau\left(1-3 x^{2}\right)\right]^{5}}\right\}\left\{\left[\frac{\sqrt{2 D b}}{a}-\frac{\tau \sqrt{2 D b}\left(1-3 x^{2}\right)}{a}\right]\right\}^{\frac{3}{2}} \\
& \cdot\left\{\begin{array}{l}
\exp \left\{\frac{1}{D} \int_{x_{1}}^{x_{2}}\left[x-x^{3}+\sqrt{\frac{b}{a^{3}}} A \cos \left(\frac{\omega_{0}}{a} t_{0}+\varphi\right)\right] \mathrm{d} x-\frac{1}{2 D} \tau\left[x-x^{3}+\sqrt{\frac{b}{a^{3}}} A \cos \left(\frac{\omega_{0}}{a} t_{0}+\varphi\right)\right]^{2}\right\} \\
+\exp \left\{\frac{1}{D} \int_{x_{1}}^{x_{2}}\left[x-x^{3}-\sqrt{\frac{b}{a^{3}}} A \cos \left(\frac{\omega_{0}}{a} t_{0}+\varphi\right)\right] \mathrm{d} x-\frac{1}{2 D} \tau\left[x-x^{3}-\sqrt{\frac{b}{a^{3}}} A \cos \left(\frac{\omega_{0}}{a} t_{0}+\varphi\right)\right]^{2}\right\}
\end{array}\right.
\end{aligned}\right.
$$

We use the maximum likelihood ratio detection, and the likelihood ratio is

$$
\begin{aligned}
& \Lambda\left(x, t_{0}\right)=\frac{P\left(x \mid \mathrm{H}_{1}, t_{0}\right)}{P\left(x \mid \mathrm{H}_{0}, t_{0}\right)} \\
& =\exp \left\{\begin{array}{c}
\frac{1}{D} \int_{-\infty}^{x}\left[\sqrt{\frac{b}{a^{3}}} A \cos \left(\frac{\omega_{0}}{a} t_{0}+\varphi\right)\right] \mathrm{d} x \\
-\frac{1}{2 D} \tau\left\{2\left(x-x^{3}\right) \sqrt{\frac{b}{a^{3}}} A \cos \left(\frac{\omega_{0}}{a} t_{0}+\varphi\right)\right\}
\end{array}\right\} \\
& +\exp \left\{\begin{array}{c}
\frac{1}{D} \int_{-\infty}^{x}\left[-\sqrt{\frac{b}{a^{3}}} A \cos \left(\frac{\omega_{0}}{a} t_{0}+\varphi\right)\right] \mathrm{d} x \\
-\frac{1}{2 D} \tau\left\{-2\left(x-x^{3}\right) \sqrt{\frac{b}{a^{3}}} A \cos \left(\frac{\omega_{0}}{a} t_{0}+\varphi\right)\right\}
\end{array}\right\}
\end{aligned}
$$

where the parameters are the same as in (10).

As described in (11), $\cos \left(\frac{\omega_{0}}{a} t_{0}+\varphi\right)$ is the linear force of the SR introduced by the DSFH signal at decision time. But due to the symmetry of the peak and valley of sine wave and the particles of SR, the effects of the positive and negative drift force are the same, not being distinguished. What is more, when the decision time is the peak or valley, the drift force reaches largest, and the pause PDF reaches largest which is best to detect.
As for different criteria, the threshold has different forms. The minimum probability or maximum posterior probability are adopted in communication systems as

$$
\Lambda\left(x, t_{0}\right) \underset{\substack{\mathrm{H}_{0} \\ \stackrel{\mathrm{H}_{1}}{\gtrless}}}{\stackrel{\mathrm{H}_{0}}{\gtrless}}
$$

where $\lambda_{0}$ is the decision threshold, and $\lambda_{0}=\frac{P_{0}}{P_{1}}=1$ for the same prior probability in communication systems.

There is no analytical solution in (12), but we can calculate the threshold numerically by (11) and (12). Next we analyze the probability of detection and false alarm. The detection probability is the probability of deciding $\mathrm{H}_{1}$ at the case $\mathrm{H}_{1}$, and the false alarm probability is the probability of deciding $\mathrm{H}_{1}$ at the case $\mathrm{H}_{0}$, which is depicted as Fig. 3 .

Owing to the symmetry of the SR particles, the probability is also symmetrical, so that the decision area also presents symmetry. As depicted in Fig. 3, the decision area of $\mathrm{H}_{1}$ is $R_{1}=\{R|R>| r \mid\}$, the decision area of $\mathrm{H}_{0}$ is $R_{0}=\{R|R \leq| r \mid\}$. So the detection probability is (13), where $\theta$ is the decision threshold, other parameters are the same as in (10).

$$
\begin{aligned}
P_{\mathrm{d}} & =\int_{R_{1}} P\left(x \mid \mathrm{H}_{1}\right) \mathrm{d} x=\int_{\theta}^{x_{2}} \rho\left(x, t_{0} \mid \mathrm{H}_{1}\right) \mathrm{d} x+\int_{x_{1}}^{-\theta} \rho\left(x, t_{0} \mid \mathrm{H}_{1}\right) \mathrm{d} x \\
& =\int_{\theta}^{x_{2}}\left\{\begin{array}{l}
N_{1} \sqrt{\frac{4 \pi D^{2} b}{a^{2} \tau\left[1-\tau\left(1-3 x^{2}\right)\right]}} \cdot\left\{1+\frac{15 D b \tau^{3} x^{2}}{a^{2}\left[1-\tau\left(1-3 x^{2}\right)\right]^{5}}\right\} \cdot\left\{\left[\frac{\sqrt{2 D b}}{a}-\frac{\tau \sqrt{2 D b}\left(1-3 x^{2}\right)}{a}\right]\right\}^{\frac{3}{2}} \\
\cdot \exp \left\{\frac{1}{D} \int_{x_{1}}^{x_{2}}\left[x-x^{3}+\sqrt{\frac{b}{a^{3}}} A \cos \left(\frac{\omega_{0}}{a} t_{0}+\varphi\right)\right] \mathrm{d} x-\frac{1}{2 D} \tau\left[x-x^{3}+\sqrt{\frac{b}{a^{3}}} A \cos \left(\frac{\omega_{0}}{a} t_{0}+\varphi\right)\right]^{2}\right\} \\
\mathrm{d} x
\end{array}\right. \\
& +\int_{x_{1}}^{-\theta}\left\{\begin{array}{l}
N_{1} \sqrt{\frac{4 \pi D^{2} b}{a^{2} \tau\left[1-\tau\left(1-3 x^{2}\right)\right]}} \cdot\left\{1+\frac{15 D b \tau^{3} x^{2}}{a^{2}\left[1-\tau\left(1-3 x^{2}\right)\right]^{5}}\right\} \cdot\left\{\left[\frac{\sqrt{2 D b}}{a}-\frac{\tau \sqrt{2 D b}\left(1-3 x^{2}\right)}{a}\right]\right\}^{\frac{3}{2}} \\
\cdot \exp \left\{\frac{1}{D} \int_{x_{1}}^{x_{2}}\left[x-x^{3}+\sqrt{\frac{b}{a^{3}}} A \cos \left(\frac{\omega_{0}}{a} t_{0}+\varphi\right)\right] \mathrm{d} x-\frac{1}{2 D} \tau\left[x-x^{3}+\sqrt{\frac{b}{a^{3}}} A \cos \left(\frac{\omega_{0}}{a} t_{0}+\varphi\right)\right]^{2}\right\}
\end{array}\right\} \mathrm{d} x
\end{aligned}
$$




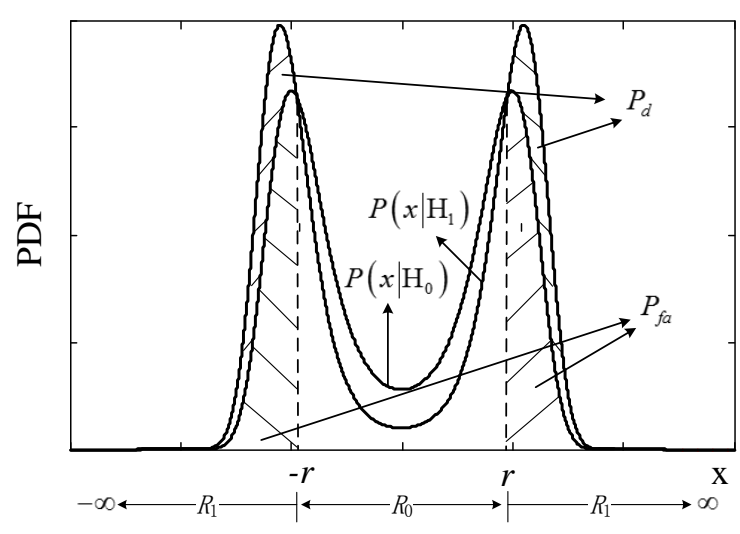

Fig. 3. The probability and region of decision.

The false alarm probability is:

$$
\begin{aligned}
& P_{\mathrm{fa}}=\int_{-\theta}^{\theta} P\left(x, \mid \mathrm{H}_{0}, t_{0}\right) \mathrm{d} x=\int_{-\theta}^{\theta} \rho\left(x, t_{0} \mid \mathrm{H}_{0}\right) \mathrm{d} x \\
& =\int_{-\theta}^{\theta}\left\{\begin{array}{c}
N_{0} \sqrt{\frac{4 \pi D^{2} b}{a^{2} \tau\left[1-\tau\left(1-3 x^{2}\right)\right]}} \cdot\left\{1+\frac{15 D b \tau^{3} x^{2}}{a^{2}\left[1-\tau\left(1-3 x^{2}\right)\right]^{5}}\right\} \\
\cdot\left\{\left[\frac{\sqrt{2 D b}}{a}-\frac{\tau \sqrt{2 D b}\left(1-3 x^{2}\right)}{a}\right]\right\}^{\frac{3}{2}} \\
\cdot \exp \left[\frac{1}{D} \int_{x_{1}}^{x_{2}}\left(x-x^{3}\right) \mathrm{d} x-\frac{1}{2 D} \tau\left(x-x^{3}\right)^{2}\right]
\end{array}\right\} \mathrm{d} x .
\end{aligned}
$$

\section{Experimental Verification}

In this section, the theory and simulation are analyzed to demonstrate the detection improvement by the Simulink model of the DSFH via SR processing. The simulation parameters are as follows: the hopping frequency segments are $30 \mathrm{kHz}-80 \mathrm{kHz}$; the sample frequency of RF is $2000 \mathrm{kHz}$; the frequency of IF is $1 \mathrm{kHz}$; the sample frequency of IF is $200 \mathrm{kHz}$.

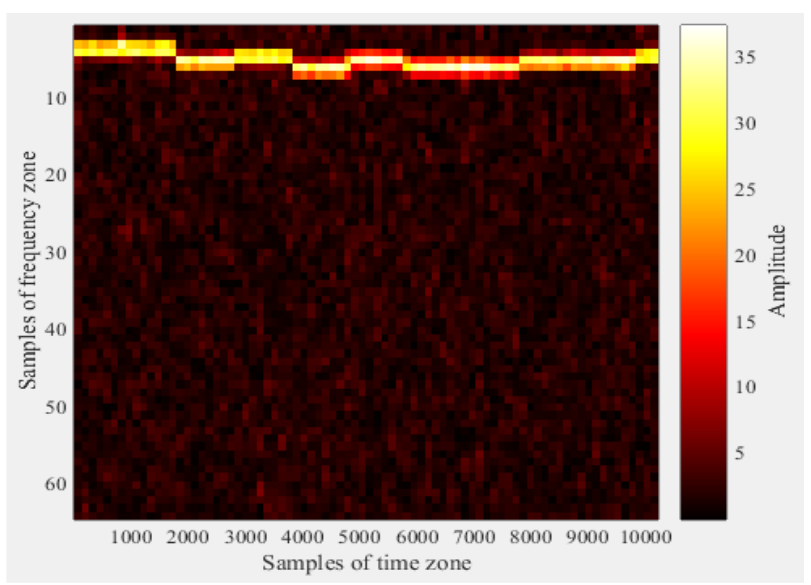

(a) Input SNR $=10 \mathrm{~dB}$.

\subsection{The Time and Frequency Feature Analysis of the DSFH Signal via SR Processing}

The time-frequency graph of the RF signal of the DSFH is described as Fig. 4. The time samples, the frequency samples and the amplitude are abscissa, ordinate and stroke coordinates respectively. There are bright hopping timefrequency spots and dark red spots of Gaussian background noise in Fig. 4(a) when SNR is $10 \mathrm{~dB}$. And there is energy leakage at the both sides of the central frequency, due to the hamming window of Short Time Fourier Transform (STFT). Owing to that the sample frequency is $2000 \mathrm{kHz}$ and that the Hamming window length is 128 , the hopping frequency spots concentrate on the upside of the positive frequency band of $1000 \mathrm{kHz}$. The time-frequency graph is filled with dark noise spots in Fig. 4(b). This is because the signal is submerged in the strong noise when input SNR is $-20 \mathrm{~dB}$. So time-frequency graph is fully filled by noise spots. That is why the traditional FH can not work well in the strong noise.

The time and frequency waveform of the IF signal received by the super-heterodyne of the DSFH signal is described as Fig. 5(a) and (b). At the same time, the time and frequency waveform of the output signal via SR processing is described as Fig. 5(c) and (d). It presents cluttered and irregular where there is no features of the sine wave of $1 \mathrm{kHz}$ in Fig. 5(a) and (b) when SNR is $-18 \mathrm{~dB}$. However it can be found periodic characteristics in the time waveform of Fig. 5(c) processed by SR, and the signal component of $1 \mathrm{kHz}$ (sample frequency is $200 \mathrm{kHz}$, and frequency resolution is 10) can be observed significantly in the frequency waveform of Fig. 5(d). What is more, the global SNR of the whole band raises $8.5521 \mathrm{~dB}$ when the output global SNR reaches $-9.4479 \mathrm{~dB}$. This is because the color noise gathers to the low frequency region leading to add the energy of this region, which pulls the particles to resonate between two wells together with the sine wave. This is equivalent to the nonlinear low pass filter which only allow the low frequency to pass.

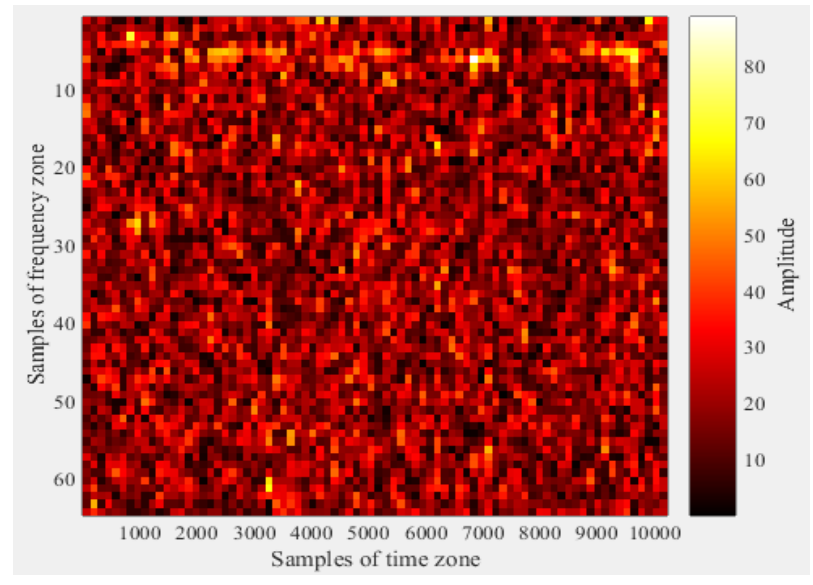

(b) Input $\mathrm{SNR}=-20 \mathrm{~dB}$

Fig. 4. The time-frequency waveform of the DSFH at different SNR. 


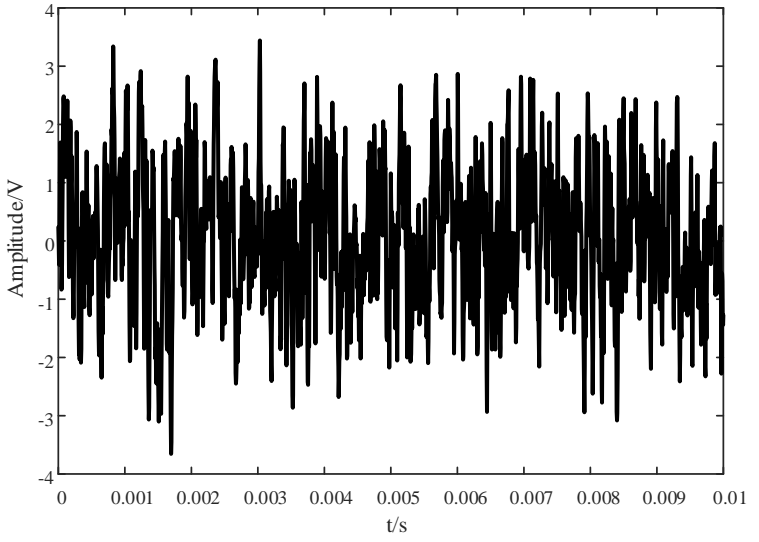

(a) The time waveform of the SR input signal.

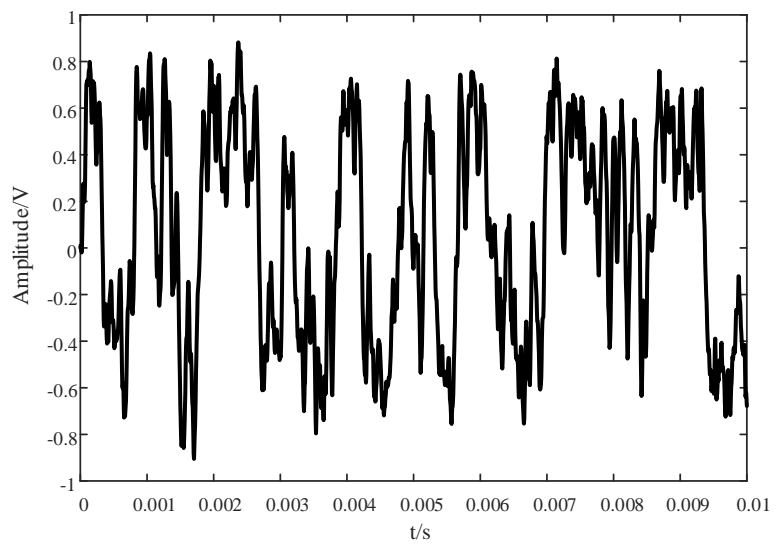

(c) The time waveform of the SR output signal.

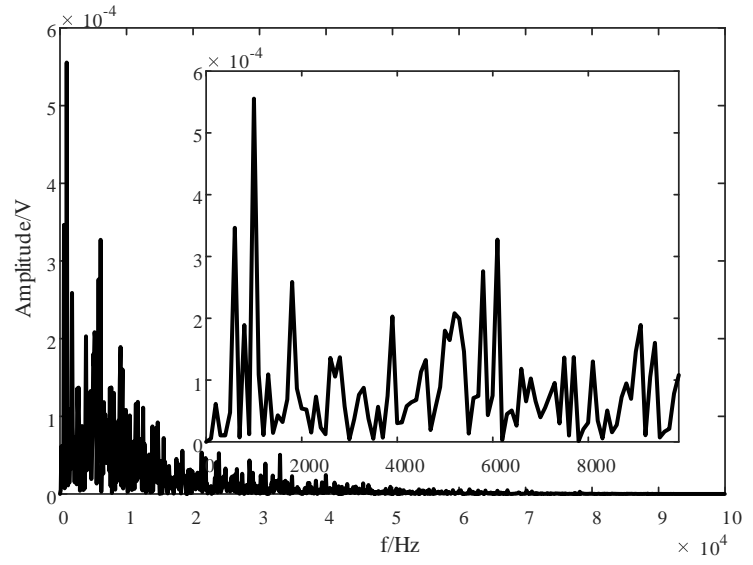

(b) The frequency waveform of the SR input signal.

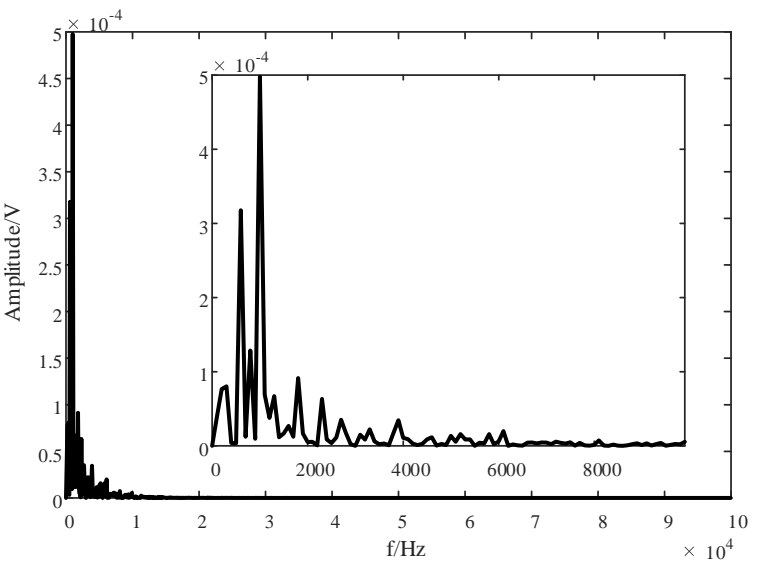

(d) The frequency waveform of the SR output signal.

Fig. 5. The time and frequency waveform of the IF signal of the DSFH via SR processing ( $\mathrm{SNR}=-18 \mathrm{~dB}, \sigma^{2}=4, A=0.25, a=1 \times 10^{4}$, $b=2.59 \times 10^{12}$ ).

So the noise of the high frequency zone will be weaken. And the signal positing the low frequency zone will be enhanced. Therefore the signal presents some degrees of periodicity in time zone, obviously in frequency zone, in which the global SNR increases in macroscopic view.

\subsection{The PDF of the DSFH Signal via SR Processing}

The theoretical and simulation results of the PDF of the IF signal of DSFH processed by SR are descripted in Fig. 6(a) and (b). It is the color noise filtered by transition bands in Fig. 6(a), and filtered by pass bands in Fig. 6(b). The force of sine wave can pull the particles to center at the both sides of wells, leading to expand the difference of the hypotheses of $\mathrm{H}_{1}$ and $\mathrm{H}_{0}$, which is useful to distinguish the existence of the sine wave of the hypotheses of $\mathrm{H}_{1}$ and $\mathrm{H}_{0}$. And the difference of the PDF under $\mathrm{H}_{1}$ and $\mathrm{H}_{0}$ is more obvious in Fig. 6(a) than in Fig. 6(b). this is because the intensity of the color noise filtered by transition bands is lower than the color noise filtered by pass bands, leading to that the local SNR is larger. What is more, the IF signal force always exists, leading to the PDF is the periodic result of the force in simulation; while the theoretical results only vary with the decision time. This is why the theoretical results differ with simulation in some degree no matter in the aspects of the steady position and the PDF.

\subsection{The Detection Performance of the DSFH Signal via SR Processing}

The ROC curves of the DSFH signal processed by SR are descripted as Fig. 7(a) and (b). Figure 7(a) depicts the case of different $\tau$ at the input $\mathrm{SNR}=-14 \mathrm{~dB}$, and Fig. 7(b) depicts the case of different SNR at $\tau=0.1$. We can see that there are different ROC curves at different SNR and different $\tau$. But all of them are the convex curves positing the left upper side of the line of $P_{\mathrm{d}}=P_{\mathrm{fa}}$ and passing the points of $(0,0)$ and $(1,1)$. What is more, the smaller the $\tau$, the bigger the $P_{\mathrm{d}}$ when noise is color, because that when the power of color noise is constant, the smaller the $\tau$, the larger the local SNR, leading better detection in Fig. 7(a). And the larger the SNR, the higher the curves. The larger the SNR, the bigger the $P_{\mathrm{d}}$ leading the better detection performance at the same $P_{\mathrm{fa}}$ in Fig. 7(a). But at the same $\tau$ and same SNR, the $P_{\mathrm{d}}$ and $P_{\text {fa }}$ decrease following the increase of the threshold $\theta$. For the 
detection of the known $\theta$, the tangency point between the curve and the line of the slope of $\theta$ is the $P_{\mathrm{d}}$ and $P_{\mathrm{fa}}$ at the current SNR and $\theta$. Therefore the ROC curve can describe the likelihood detection performance of the DSFH signal via SR processing wholly.

The detection performance of the DSFH signal via SR processing varies with SNR is described as Fig. 8. The theoretical and simulation results of $P_{\mathrm{d}}$ increase with the SNR increase. This is because the increase of SNR can raise the detection performance. The theoretical detection performance of $\tau=0.0035$ is better than that of $\tau=0.1$, it is because when the power intensity of the color noise is constant, it distributes uniformly during the low frequency band $(|\omega| \ll 1 / \tau)$, leading to the larger local SNR than that of $\tau=0.1$. What is more, the IF signal locates in the transition band of the filter corresponding to $\tau=0.0035$, leading to higher local SNR than that of the pass band of the filter corresponding to $\tau=0.1$ in simulation. So the signal component occupies more percent leading to bigger $P_{\mathrm{d}}$. At the same time, there is some difference between theoretical and simulation results at the two cases, which is more obvious at lower SNR, that is because the value of $P_{\mathrm{d}}$ can not be obtained in simulation where the value of $P_{\mathrm{e}}$ can be obtained. And their relationship is $P_{\mathrm{e}}=\frac{1}{2}\left(1-P_{\mathrm{d}}+P_{\mathrm{fa}}\right)$. The $P_{\mathrm{d}}=1-P_{\mathrm{e}}$ can be tenable at high SNR due to that the $P_{\mathrm{fa}}=1-P_{\mathrm{d}}$ is tenable with that the difference of PDF between the hypotheses of $\mathrm{H}_{1}$ and $\mathrm{H}_{0}$ is small. But the difference are big at low SNR, so the equation of $P_{\mathrm{d}}=1-P_{\mathrm{e}}$ is not tenable, causing the difference between the theory and simulation. And the $P_{\mathrm{e}}$ is always smaller than 0.5 (random decision without any receptive process), so the $P_{\mathrm{d}}$ is always bigger than 0.5 in simulation.
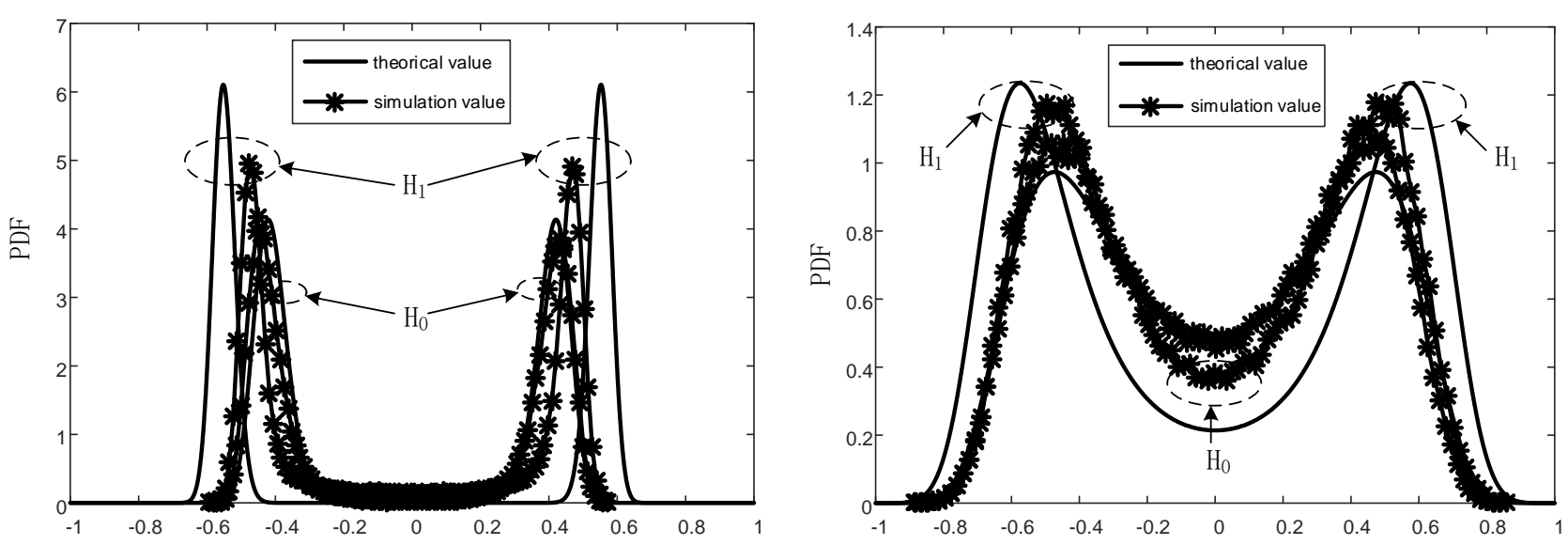

(a) $\tau=0.0035$.

(b) $\tau=0.1$.

Fig. 6. The PDF of the particles at different position $\left(\mathrm{SNR}=-14 \mathrm{~dB}, \sigma^{2}=4, A=0.25, a=1 \times 10^{4}, b=5.65 \times 10^{12}\right)$.

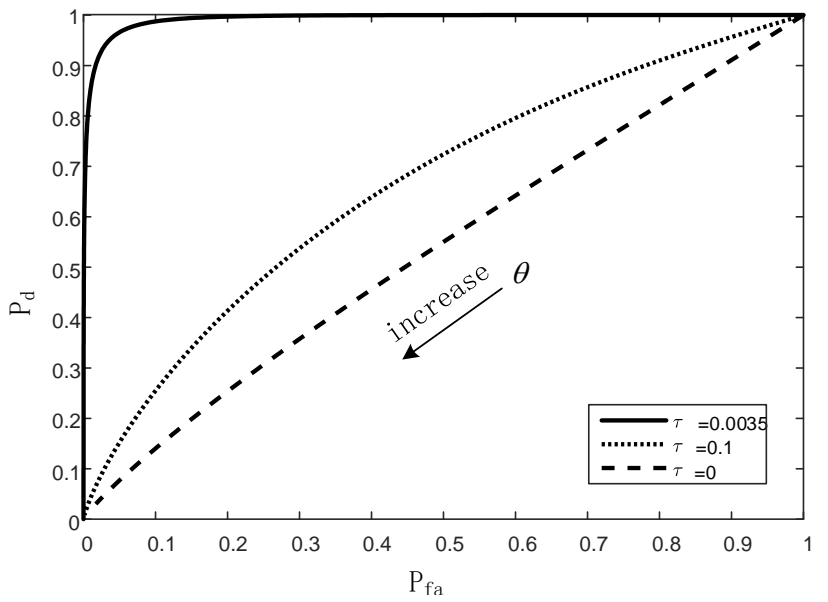

(a) Different $\tau$ when $\mathrm{SNR}=-14 \mathrm{~dB}$.

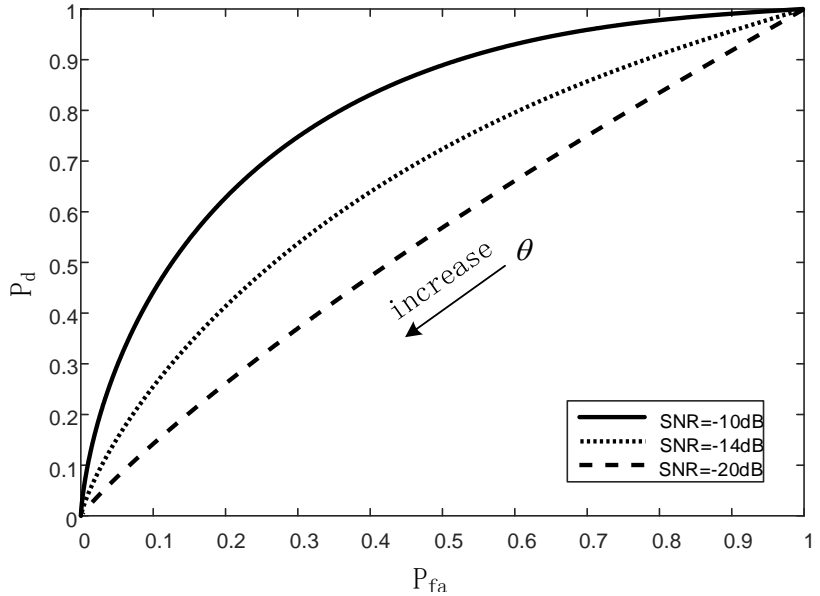

(b) Different SNR when $\tau=0.1$.

Fig. 7. The ROC curves of the DSFH via SR processing. 


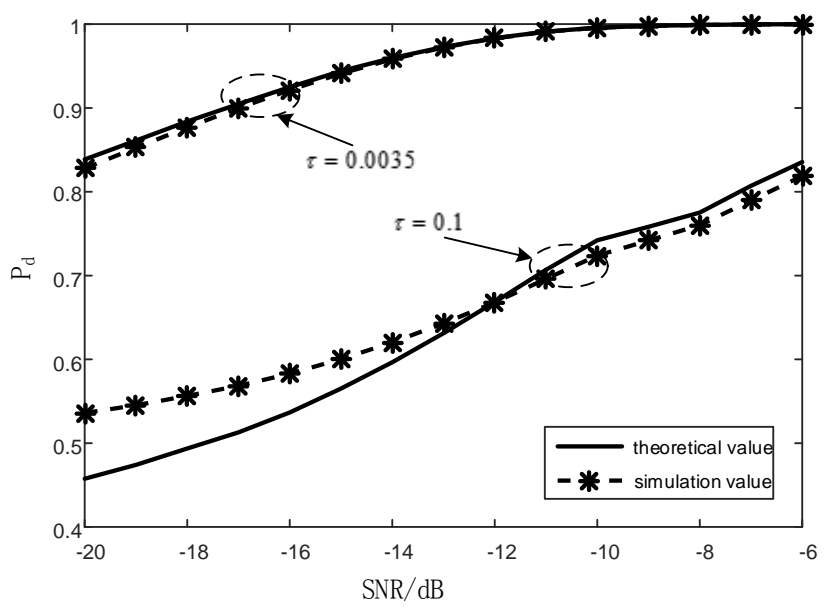

Fig. 8. The detection performance of the DSFH via SR processing.

\section{Conclusion}

By analyzing the physical characteristics of the DSFH signal, we apply the SR to the DSFH mode. The signal models of transmission, reception and the IF of the DSFH are constructed. And the scale transaction is used to adjust the IF signal to fit the SR. But due to the color noise, the LE is non-markovian, so we expand the 1-D LE to a 2-D one which is markovian. Then the non-autonomous FPE is transformed into an autonomous one by assuming that the SR transition of magnetic particles is instantaneous and introducing the decision time. Therefore, the analytical periodic steady solution of the PDF with the parameter of the correlation time is obtained. Finally, the detection probability, false alarm probability and the ROC curve are obtained under the criterion of MAP. Through the theoretical and simulation results, we conclude that 1) whether the DSFH can work under strong color noise is decided by the correlation time of the color noise; 2) when the power intensity of the color noise is constant, the smaller the correlation time with the bigger local SNR, the greater PDF difference of the SR output under two hypothesis, leading to better detection performance. This conclusion provides theoretical support for the DSFH works under color noise at extremely low SNR, and the FPE solution of introducing the decision time can inspire other FPE solutions.

\section{References}

[1] FITZEK, F. H. P. The medium is the message. In Proceedings of the IEEE International Conference on Communications (ICC). Istanbul (Turkey), 2006, p. 5016-5021. DOI: 10.1109/ICC.2006.255461

[2] ZHOU, X., KYRITSI, P., EGGERS, P. C. F. The medium is the message: Secure communication via waveform coding in MIMO systems. In Proceedings of the IEEE Vehicular Technology Conference (VTC). Dublin (Ireland), 2007, p. 491-495. DOI: 10.1109/VETECS.2007.112
[3] QUAN, H., ZHAO, H., CUI, P. Anti-jamming frequency hopping system using multiple hopping patterns. Wireless Personal Communications, 2015, vol. 81, no. 3, p. 1159-1176. ISSN: 0929-6212. DOI: $10.1007 / \mathrm{s} 11277-014-2177-1$

[4] ZHAO, H., QUAN, H.-D., CUI, P.-Z. Follower-jamming resistible multi-sequence frequency hopping wireless communication. Systems Engineering and Electronics, 2015, vol. 66, no. 3, p. 671-678. ISSN: 1001-506X. DOI: 10.3969/j.issn.1001-506X.2015.03.31 (in Chinese)

[5] DU, C., QUAN, H., CUI, P., et al. Carrier sense random packet CDMA protocol in dual-channel networks. Radioengineering, 2015, vol. 24, no. 2 , p. 507-517. ISSN: $1210-2512$. DOI: $10.13164 /$ re.2015.0507

[6] BENZI, R., SUTERA, A., VULPIANI, A. The mechanism of stochastic resonance. Journal of Physics A: Mathematical and General, 1981, vol. 14, no. 11, p. 453-457. ISSN: 1751-8113. DOI: $10.1088 / 0305-4470 / 14 / 11 / 006$

[7] ZHANG, G., SONG, Y., ZHANG, T.-G. Characteristic analysis of exponential type mono-stable stochastic resonance under Levy noise. Journal of Electronics \& Information Technology, 2017, no. 4, p. 893-900. ISSN: 1009-5896. DOI: 10.11999/JEIT160579 (in Chinese)

[8] WANG, S., WANG, F.-Z. Adaptive stochastic resonance system in terahertz radar signal detection. Acta Physica Sinica, 2018, vol. 67, no. 16, p. 1-7. ISSN: 1000-3290. DOI: 10.7498/aps.67.20172367 (in Chinese)

[9] KRAUSS, P., METZNER, C., SCHILlinG, A. Adaptive stochastic resonance for unknown and variable input signals. Scientific Reports, 2017, vol. 7, no. 1, p. 1-8. ISSN: 2045-2322. DOI: $10.1038 / \mathrm{s} 41598-017-02644-\mathrm{w}$

[10] HANGGI, P., JUNG, P., ZERBE, C., et al. Can colored noise improve stochastic resonance? Journal of Statistical Physics, 1993, vol. 7, no. 1, p. 25-47. ISSN: 0022-4715. DOI: 10.1007/bf01053952

[11] FUENTES, M. A., TORAL, R., WIO, H. S. Enhancement of stochastic resonance: The role of non Gaussian noises. Physica A, 2001, vol. 295, no. 1, p. 114-122. ISSN: 0378-4371. DOI: $10.1016 / \mathrm{s} 0378-4371(01) 00062-0$

[12] FUENTES, M. A., WIO, H. S., TORAL, R. Effective Markovian approximation for non-Gaussian noises: A path integral approach. Physica A, 2002, vol. 303, no. 1, p. 91-104. ISSN: 0378-4371. DOI: $10.1016 / \mathrm{s} 0378-4371(01) 00435-6$

[13] JIA, Y. , YU, S. N. , LI, J. R. Stochastic resonance in a bistable system subject to multiplicative and additive noise. Physical Review E, 2000, vol. 62, no. 2, p. 1869-1878. ISSN: 1539-3755. DOI: 10.1103/PhysRevE.62.1869

[14] XU, B. , LI, J., DUAN, F., et al. Effects of colored noise on multifrequency signal processing via stochastic resonance with tuning system parameters. Chaos, Solitons and Fractals, 2003, vol. 16, no. 1, p. 93-106. ISSN: 0960-0779. DOI: 10.1016/s0960-0779(02)00201-1

[15] HU, N.-G. Theory and Method of Detecting Weak Characteristic Signals of Stochastic Resonance. Beijing (China): National Defense Industry Press, 2012. p. 85-86. ISBN: 9787118081558 (in Chiness)

[16] HU, G. Stochastic Forces and Nonlinear Systems. Shanghai (China): Shanghai Scientific and Technological Education Publishing House, 1994. p. 184-208, 222-232. ISBN: $7542808931 / O .53$ (in Chiness)

\section{About the Authors ...}

Guangkai LIU was born in Shijiazhuang, China in November 1990 . He received his B.E. degree and M.E. degree from Army Engineering University in 2013 and 2015. His research 
interests include military anti-jamming communication and weak signal detection.

Yanmei KANG was born in Henan, China in December 1973. She received her PhD. degree from Xi'an Jiaotong University in 2004. Her research interests include applied stochastic dynamical systems and probalistic and statistical methods in machine learning.

Houde QUAN was born in Dalian, China in 1963. He received his $\mathrm{PhD}$. degree from Northwestern Polytechnical University, China in 2008. His research interests include military anti-jamming communication.
Huixian SUN was born in Baotou, China in 1980. He received his M.E. degree and $\mathrm{PhD}$. degree from National University of Defense Technology respectively in 2005 and 2010.

Peizhang CUI was born in Datong, China in 1974. He received his M.E. degree from the 54 Electrical Science Institutions in China in 2003. His research interests include military anti-jamming communication.

Chao GUO was born in Hubei, China in 1991. He received his M.E. degree from Army Engineering University in 2016. His research interests include military anti-jamming communication. 